\title{
Context-dependent factored language models
}

\author{
Gregor Donaj* and Zdravko Kačič
}

\begin{abstract}
The incorporation of grammatical information into speech recognition systems is often used to increase performance in morphologically rich languages. However, this introduces demands for sufficiently large training corpora and proper methods of using the additional information. In this paper, we present a method for building factored language models that use data obtained by morphosyntactic tagging. The models use only relevant factors that help to increase performance and ignore data from other factors, thus also reducing the need for large morphosyntactically tagged training corpora. Which data is relevant is determined at run-time, based on the current text segment being estimated, i.e., the context. We show that using a context-dependent model in a two-pass recognition algorithm, the overall speech recognition accuracy in a Broadcast News application improved by $1.73 \%$ relatively, while simpler models using the same data achieved only $0.07 \%$ improvement. We also present a more detailed error analysis based on lexical features, comparing first-pass and second-pass results.
\end{abstract}

Keywords: Speech recognition, Factored language model, Dynamic backoff path, Word context, Inflectional language, Morphosyntactic tags

\section{Introduction}

Speech recognition still performs poorly in inflectional languages compared to mainstream languages like English. The cause can be found in the rich morphology of such languages, which increases the need for larger vocabularies. It is estimated that inflectional languages need up to ten times larger vocabularies than English $[1,2]$.

Although we can easily build larger vocabularies, thus reducing the number of recognition errors caused by outof-vocabulary words, the use of such models increases not only the size of language models but also the required size of training corpora. With larger vocabularies, words are also substituted more easily, especially if they are acoustically similar. Many errors occur only in the grammatical sense, when a recognition error results in a word with the same lemma but with a grammatical error, e.g., a false case or number.

An often used approach in speech recognition of morphologically rich languages is the incorporation of grammatical information in some form. One practical implementation to achieve this is the use of a factored language

*Correspondence: gregor.donaj@um.si

Faculty of Electrical Engineering and Computer Science, University of Maribor, Smetanova ul. 17, SI-2000, Maribor, Slovenia model (FLM). Such models were first proposed for speech recognition in Arabic languages [3], but they have also been adopted in statistical machine translation [4] and, more recently, in natural language generation [5].

In an FLM, each word is represented as a vector of factors containing information about the word. One of the factors is usually the word itself; other factors can be the lemma, the morphosyntactic description (MSD) tag, the word stem, the ending, the root, etc. While traditional (word-based) $n$-gram language models use only the basic word forms to estimate the probabilities of sentences, FLMs can use all defined factors and their information. Since these factors have fewer possible values (smaller vocabularies), we can build simple models on individual factors using smaller training corpora.

The idea of FLMs, however, is to use different factors in the same models. As each word can have several factors, such an FLM will have many more terms in its conditional probability than traditional $n$-gram models or an FLM using only one factor. The potentially large number of selected factors again increases the need for larger training corpora, which may not be available for some languages or applications, causing data sparsity problems. 
The two important steps for defining an FLM are to define a set of relevant factors and to define the backoff path, which determines the order in which to discard factors in the case of training data sparsity.

In the present paper, we propose a novel approach to factor definition and backoff path selection for FLMs, in which the model considers only specific factors from specific words that can improve recognition performance. The selection of these factors and their order in the backoff path is made dynamically, based on the parts of speech of words in the current context. Thus, the name is context-dependent FLMs. With this approach, we can build FLMs with a limited number of factors in each probability estimation, which can improve recognition performance while avoiding new data sparsity problems. This method also makes use of grammatical properties of the target language, as the process of determining the backoff path searches for specific correlations in a given sentence structure.

\subsection{Previous work}

The first results on FLMs were perplexity calculations, e.g., on the Arabic Callhome corpus [6] or the Wall Street Journal corpus [7]. In both studies, the authors reported perplexity improvements. Later, the models were used in speech recognition applications [3].

While a backoff path can be selected manually and may remain fixed, the authors of FLMs proposed several methods for determining the backoff path based on training data, e.g., the (normalized) number of occurrences and the maximum probability of the vertex. One of these methods- $\min / \max$ counts - was used by the authors in [8]. The authors in [7] also proposed to using several backoff paths simultaneously and determining the set of those paths in run-time.

FLMs are often used in Arabic and Slavic languages, probably most prominently in Russian $[9,10]$, as well as in other morphologically rich languages such as Romanian [8], Turkish [11], and Amharic [12].

There is previous work with $N$-best list rescoring using FLMs. In [13], the authors presented an $N$-best list rescoring approach in which the language model was adapted based on the vocabulary in the hypothesis list. While the system used morpheme-based models in the first pass, the performance improved in the second pass, in which the models were word based. Part of the difference between the first and second pass can be explained with the use of trigram sub-wordbased models in the first pass and the trigram wordbased models in the second pass. It was shown that sub-word-based models require higher model orders to achieve performance comparable with word-based models [14]. The authors in [15] implemented FLMs with the use of morpheme units for language modeling. With
$N$-best list rescoring, they achieved an improvement of $0.3 \%$ absolutely.

Sak [11] showed for the Turkish language that the improvements achieved by using FLMs rather than traditional $n$-gram models are greater while only limited size corpora are available for training; the improvements decreased as the corpus size increased. These results can be considered relevant to speech recognition in specific domains with limited training data, where data sparsity becomes a problem for word-based models.

In [16], the authors defined trigger part-of-speech (POS) tags and used them as syntactic features, demonstrating that FLMs can outperform traditional trigram language models regarding perplexity and mixed error rate in speech recognition. While their approach included POS tags, the backoff paths were selected based on overall perplexity.

In previous research, backoff path selection is often made with fixed backoff paths, a method based on $n$-gram occurrences in training data, or overall perplexity results on development data. Our approach differs from previous work as we perform backoff path selection for each word sequence individually in run-time, selecting the path differently for each possible sequence of POS tags in the context and basing the decision on the perplexity results relevant only to the considered sequence of POS tags.

\subsection{Structure of the paper}

In Section 2, we present the basics of FLMs required for an understanding of the proposed context-dependent FLMs and their implementation. In Section 3, we present our proposed models along with all of the algorithms necessary to efficiently determine backoff path selection, and, in Section 4, a two-pass recognition algorithm suitable for using the proposed models is introduced. In Section 5, we describe our experimental system used for building and testing the models. The obtained results are then presented in Section 6, followed by the conclusion in Section 7.

\section{Morphosyntactic description tags}

A basic process of text or speech annotation is the addition of part-of-speech (POS) tags to each word in a text. The most basic tags would be noun, adjective, verb, adverb ...residual. However, to be more useful, typically additional properties are considered. Tags which hold more information, especially in morphologically rich languages, are called morphosyntactic description (MDS) tags.

For example, Penn Treebank Project specifies 36 different POS tags for the English language. Some part of speech have several possible tags, e.g., there are four different nouns listed with tags in parentheses:

- Noun, singular or mass (NN)

- Noun, plural (NNS) 
- Proper noun, singular (NP)

- Proper noun, plural (NPS)

These tags are used by TreeTagger, a freely available MSD tagger for several languages.

As English is not a morphologically complex language, its POS tags give only sparse information on the grammatical role of a word in a sentence. Typically, information that is given by POS tags is the number for nouns (singular or plural) and some information about verbs (base form, past tense, present participle, third person, etc.).

In the MULTEXT-east project [17], tagsets for several languages were specified and standardized including a more complex tagset for English. Version 5 of the project specifies 16 categories (parts of speech) with a total of 31 attributes and a set of 135 different tags. For example, verbs have the following attributes: type, verb form, tense, person, and number, and the attribute person has the following possible values: first, second, and third.

Slovene, being morphologically complex language, has much more different tags. The JOS specifications [18], which were derived from the MULTEXT-east system, define 12 parts of speech. Again, some parts of speech have several attributes, e.g., verbs have the following attributes: type, aspect, form, person, number, gender, and negative. Some parts of speech have none additional attributes, e.g., particles and interjections. JOS specifies 12 parts of speech with a total of 37 attributes and a set of 1902 different tags.

Considering all possible values for all attributes of a given part of speech and all parts of speech, JOS defines 1902 different MSD tags for Slovene.

Figure 1 shows a Slovene sentence with part of speech tags. The set was annotated with Obeliks, a tagger for Slovene. The text is in XML-TEI format and is also tagged with lemmas. A translation of the sentence into English is tagged in Fig. 2. The translation was tagged with TreeTagger and result converted into the same XML-TEI format. The sentence was taken from the BNSI test set, later used in this research.

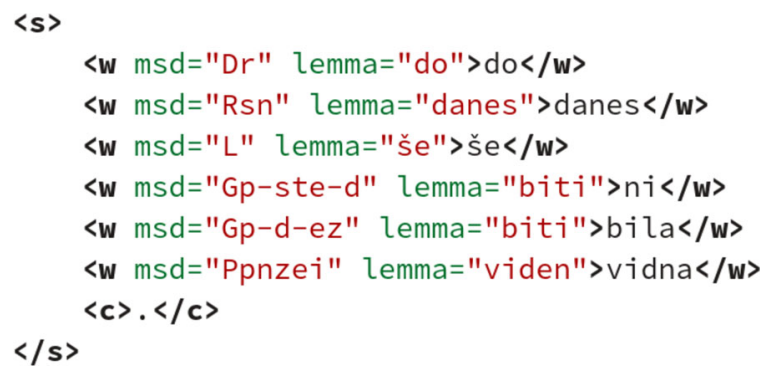

Fig. 1 An MSD-annotated text in Slovene

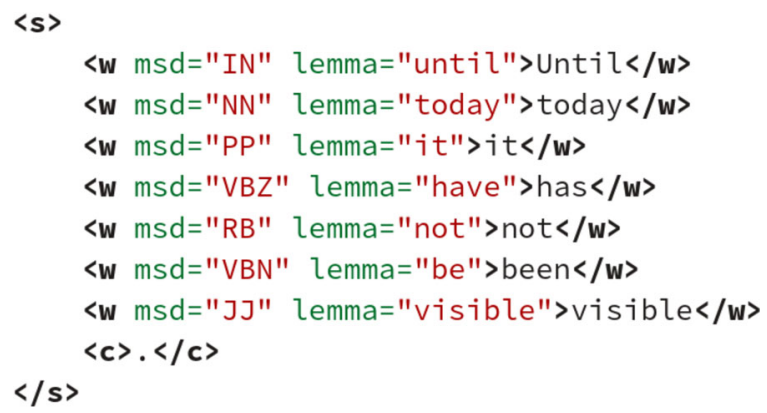

Fig. 2 An MSD-annotated text in English

\section{Factored language models}

Language models are used to estimate the probability of a given word sequence $W=\left(w_{1}, w_{2}, \ldots, w_{n}\right)$. Most commonly used are traditional word-based $n$-gram language models, which estimate the conditional probability of each word given the previous $n-1$ words. The current and previous words in these models are often called the context.

In an FLM, each word $w$ is represented as a vector of $K$ factors $w_{i}=\left(f_{i}^{1}, f_{i}^{2}, \ldots, f_{i}^{K}\right)$, where $i$ is the index number of the word. Much like in word-based $n$-gram language models, the probability of a sentence is the product of the probabilities of individual words. In the case of an FLM, we use the conditional probabilities of all factors in the estimated word given the factors of previous words:

$$
P\left(f_{i}^{1: K} \mid f_{i-1}^{1: K}, f_{i-2}^{1: K}, \ldots, f_{i-n+1}^{1: K}\right) .
$$

Using the chain rule for probabilities, we can express this probability with

$$
P\left(f_{i}^{1: K}\right)=\prod_{k=1}^{K} P\left(f_{i}^{k} f_{i}^{1: k-1}, f_{i-1}^{1: K}, \ldots, f_{i-n+1}^{1: K}\right) .
$$

The probabilities of individual factors on the right-hand side are again conditional probabilities given the factors from previous words and previous factors from the current word. With several words of history and several factors per word, the total number of given factors can quickly exceed a feasible amount for practical application.

Similarly, in word-based $n$-gram models, the above equation also makes use of data from sequences of $n$ words so that we can speak of an $n$-gram FLM. We say that $n$ is the FLM order. We also use this definition of the FLM order when only some of the possible factors are present in the conditional probability.

In the above equations, probabilities are estimated statistically. Due to data sparsity, we cannot always efficiently determine probability estimates in language models. Both word-based models and FLMs employ a technique called backoff [19], in which we discard terms in the conditional 
probability and back off to a model with fewer terms. If necessary, we can repeat the process to reduce the number of terms further. The selection of a backoff path in a word-based $n$-gram model is rather clear: on each step, we discard the most distant word, based on the assumption that more distant words have less impact on the current word. However, in an FLM, several factors can have the same distance from the current factor. Furthermore, there is no guarantee that discarding one of the most distant factors will result in better performance than discarding some closer factor, whether it is the same type of factor or not.

Often, lemmatizers and MSD taggers are used as tools to automatically determine grammatical properties. In this case, it is important that we try to identify all of the factors with the potential to improve performance in the application and include only those factors in the model. While other factors may not decrease performance, they will increase the size of the model and make the final application more time and space consuming.

In the context of FLMs, the backoff path first became relevant and the backoff graph was introduced. We can define the backoff path as a sequence of factor subsets. On every step in the backoff procedure, we move to another subset that has one factor less than the previous subset. The selection of the factor to be removed at each step determines the backoff path. Assuming we have $k$ factors as the conditional terms in the FLM probability, we start with the set of all factors. On every step, we discard one factor that is still in the path. This method gives us $k$ ! possible backoff paths. Figure 3 shows a backoff graph that illustrates this principle in the case of three factors. The vertices of the directed graph represent subsets of factors used in the conditional probability.

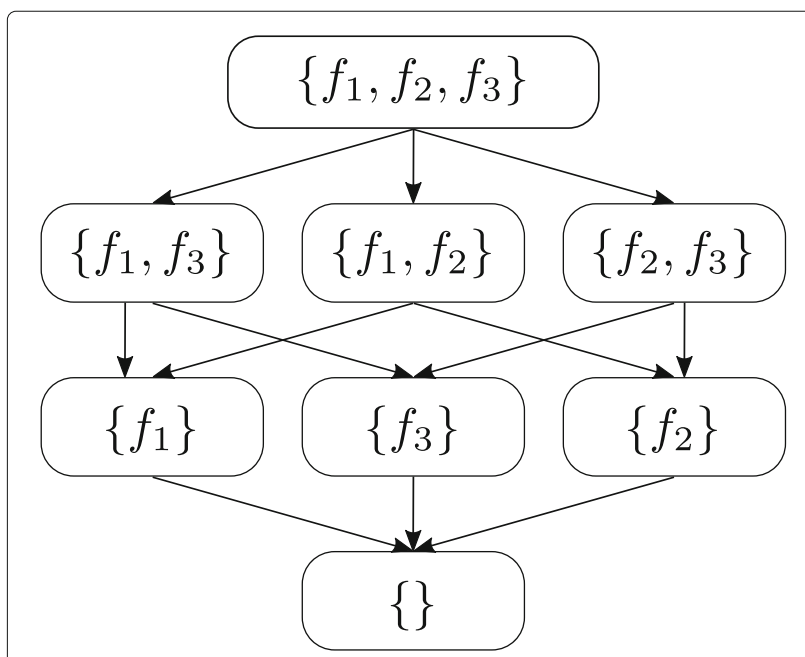

Fig. 3 Backoff graph for an FLM with three factors

\section{The context-dependent backoff path}

In the proposed models, we will use run-time determination of the backoff path, although we will use only one path simultaneously. Backoff path determination will be achieved using POS information from the currently estimated word sequence. We have therefore called these backoff paths and models context-dependent. We will later define the backoff function, which will tell us which path to use for probability estimation, given the current word and its context.

We based the proposed models on the property of grammatical matching in inflectional languages, in which the properties of some words correlate with the properties of other words. An example is the matching of case, number, and gender between nouns and adjectives. Given that these words might not be adjacent in the sentence, we made the assumption that it is not always best to discard more distant factors; examining which parts of speech are present in the sentence might suggest which factors are better suited for probability estimation.

The first step in building the proposed language models is to determine the set of different factors for each word. We found that word-based $n$-gram language models still have the greatest impact on performance. Thus, one factor shall remain the word itself. Other factors can be the lemma and grammatical properties, either as separated factors or combined (e.g., MSD tag). We can also include other information if it is useful and if appropriate tools are available to determine it automatically.

One necessary piece of information in the proposed models is the POS tag, which can be incorporated as a separate factor or can be included within the MSD tag.

\subsection{The backoff function}

Let $P_{i}$ denote the POS tag of word $w_{i}$. Assuming we have an input word sequence $\left(w_{i-n+1}, \ldots, w_{i}\right)$, we can determine a corresponding POS sequence $\left(P_{i-n+1}, \ldots, P_{i}\right)$. We then define the backoff function

$$
\text { BO : }\left(P_{i-n+1}, \ldots, P_{i}\right) \mapsto\left(f_{1}, \ldots, f_{l}\right),
$$

for every possible POS sequence, where $\left(f_{1}, \ldots, f_{l}\right)$ is an ordered set of factors to be included in the conditional probability (Eq. 2). While the set alone defines the starting point in the backoff graph, its order determines the backoff path. Considering the above equation, we will start with $l$ factors and then remove them one by one from right to left.

Let us assume that for each word, we have defined $K$ factors and we are considering a model of order $n$. The conditional probability will have between $K \cdot(n-1)$ and $K \cdot(n-1)+K-1$ possible conditional factors depending on the modeled factor. Let $|P|$ denote the number of all possible values of POS tags including start-of-sentence 
and stop-of-sentence tags. We can derive the total number of valid POS sequences of length $n \geq 2$ as:

$$
(|P|-1)^{2} \cdot(|P|-2)^{n-2} \text {. }
$$

For a sequence to be valid, the start-of-sentence tag can appear only in the first place and the end-of-sentence tag can appear only in the last place, while all other tags are not restricted.

Factor probabilities can be expressed conditionally given the factors of previous words and the previous factors of the current word. If we are currently considering factor $k$, the number of possible conditional factors $m$ is

$$
m=(n-1) \cdot K+k-1 .
$$

If we were to look for backoff paths that start with a set of all possible factors, the number of backoff paths would be $m$ !. For shorter paths of length $l$, the number of possible backoff paths is

$$
\frac{m !}{(m-l) !} \text {. }
$$

Finally, the number of paths of all possible lengths is

$$
\sum_{l=0}^{m}\left(\frac{m !}{(m-l) !}\right)
$$

where we also include a path of length 0 , which is a model containing only the modeled factor itself and no conditional factors.

Depending on the number of possible POS tags and the model order, the number of different POS sequences can increase beyond a feasible amount. The number of all possible paths increases even faster, exceeding $10^{8}$ for trigram models with three factors, for example. Therefore, it is necessary to implement an efficient method to determine the backoff function BO.

\subsection{Initial algorithm}

Let us first assume that we have the necessary equipment to consider the whole search space and a training set large enough to train all models. A simple brute-force search to determine backoff paths is presented in Algorithm 1, where $N$ is the maximal model order, $K$ is the number of factors in a word, and $\mathcal{P}_{n}$ is the set of all possible POS sequences of length $n$.

The final output of the algorithm is one backoff path $\hat{M}$ for each possible POS sequence, given the model order and the factor to be estimated. The algorithm works by testing all possible paths $\mathcal{M}$ and selecting the path that maximizes a certain function $T$. We need to repeat the algorithm for different model orders and all defined factors.

Let us consider the computational complexity of the initial algorithm. The maximum model order $N$ and the number of factors per word $K$ are given. The number of

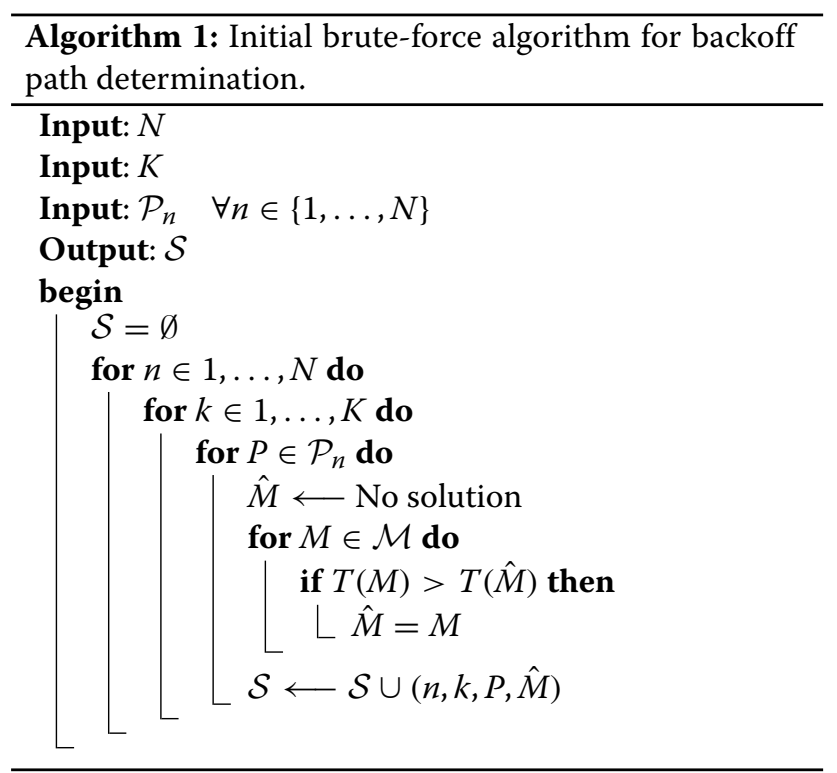

possible POS sequences $\mathcal{P}_{n}$ is given in Eq. 4, as a function of the number of different POS tags $|P|$. The number of all paths of the maximum given length $l$ is given in Eq. 7. Considering the nested loops in Algorithm 1, the total number of models to be trained and tested can be expressed as

$$
\sum_{n=1}^{N}\left(\sum_{k=1}^{K}\left((|P|-1)^{2}(|P|-2)^{n-2} \frac{m !}{(m-l) !}\right)\right),
$$

where $m$ is described in Eq. 5 .

The number of possible POS sequences can be ignored when we are interested in the umber of models to be trained and tested, since models can be reused for any POS sequence, and results can be filtered accordingly to the actual POS sequence. In any case, we can then derive the computational complexity of the brute-force algorithm as

$$
\mathcal{O}\left(|P|^{N}(N K)^{(N K)}\right) .
$$

Clearly, this algorithm is not tractable and we shall, therefore, derive a feasible system.

However, the presented algorithm will be the starting point from which we will derive a feasible system. In order to achieve this, we have to address several issues

1. Determine a criterion function $T$ that will be fast and will have the ability to estimate the performance of models. The criterion function is needed to estimate which model will perform better in the final application.

2. Define a reasonably ordered list of paths to be tested, from which we can quickly obtain a satisfactory result along with criteria determining when to discontinue 
searching. Such a list is essential as a search through all possible paths would not be feasible.

3. Define a method to combine different POS sequences, as the training data for some individual sequences would be too small for obtaining a reliable probability estimate.

\subsection{Criterion function}

In order to determine a language model's performance, we could use it in the final application, in our case speech recognition. This will provide the best estimate of whether a particular model will outperform another model or not. This method is only feasible if we test just a handful of models. However, this does not apply to our case.

We often estimate the performance of a model by calculating its perplexity on a selected development text. Compared to estimation within speech recognition, this method is much faster, as we only need to estimate each sentence once. Furthermore, it has been shown that there is a correlation between a language model's perplexity and its performance in speech recognition [20].

In the presented algorithm, we want to compare models with different backoff paths. While the perplexity of a model is typically estimated using the whole text, we now want this estimate only at words with the given context.

The backoff path selection for each word will be independent of the backoff path selection at neighboring words and so the contributions of individual words to the perplexity of the whole text will also be independent. Thus, we can use perplexity and slightly modify its calculation for use as our criterion function. This modification will be necessary as we need to compare models not on the whole text but only at words with a given context.

The perplexity PP of a model $M$ on a text $\mathcal{W}$ is defined by

$$
\operatorname{PP}_{M}(\mathcal{W})=2^{H_{M}(\mathcal{W})}
$$

where $H_{M}(W)$ is the cross entropy

$$
\begin{aligned}
H_{P}(\mathcal{W}) & =\frac{1}{-T} \cdot \log _{2} P(\mathcal{W}) \\
& =\frac{1}{-T} \cdot \log _{2} \prod_{i=1}^{T} P\left(w_{i}\right)
\end{aligned}
$$

where $T$ is the total length of the text.

We want to modify the perplexity definition to our needs. Let $P^{*}$ denotes a given POS sequence. For each $P^{*}$, we define a set of numbers

$$
\mathcal{I}\left(P^{*}\right) \subset\{1, \ldots, T\},
$$

containing indexes of words with the POS context $P^{*}$ :

$$
i \in \mathcal{I}\left(P^{*}\right) \Longleftrightarrow\left(P_{i-n}, \ldots, P_{i}\right)=P^{*} .
$$

We can then define a modified cross entropy on the subset of corresponding words as

$$
H^{*}\left(\mathcal{W}, P^{*}\right)=\frac{1}{-\left|\mathcal{I}\left(P^{*}\right)\right|} \log _{2} \prod_{i \in \mathcal{I}\left(P^{*}\right)} P\left(w_{i}\right) .
$$

For our criterion function, we can then use the modified perplexity

$$
P P^{*}\left(\mathcal{W}, P^{*}\right)=2^{H^{*}\left(\mathcal{W}, P^{*}\right)} .
$$

We shall note that better models have a lower perplexity, and we can decide either to minimise the criterion function or to use the negative value of the perplexity as a criterion function $T$ and maximize it. In the present paper, we will use the latter approach.

\subsection{Backoff path lists}

We derived a search algorithm that will build a list of backoff paths to be tested considering the following guidelines:

1. Up to a certain length, it is still feasible to test all possible paths, and we shall do so. We can build longer paths by extending the best shorter paths.

2. We will use a beam width, a technique used by speech recognition search algorithms to exclude less likely hypotheses. As in these recognition search algorithms, we will keep a list of possible paths and exclude candidates that have results (obtained by the criterion function) that fall sufficiently below the current best path.

3. If a certain path is given, we will search for additional factors considering the factors from the next word back not only in the current word's history but also in several words of history.

The backoff path search Algorithm 2 takes the current list as input and gives a new list of backoff paths as output. For each path in the input list, the algorithm determines all possible factors $\mathcal{F}^{*}$ with which the path can be expanded. After we include these new paths in the list, the algorithm tests them with the criterion function and finds the best backoff path $\mathcal{M}^{*}$. Finally, a beam width $\varepsilon$ is determined and all paths falling outside the beam are discarded.

The search algorithm must be repeated $m$-times, where $m$ is the maximum number of factors allowed in a path, as the algorithm always creates paths that are one factor longer than the input paths. However, the algorithm is not employed for short paths, as the list is generated with all possible paths. The final result is a list of backoff paths of length $m$ or shorter.

The beam width $\varepsilon$ is empirically determined for each length of the path. In our experimental system, we found that for shorter paths, $\varepsilon$ shall be greater. We saw that in shorter paths, the addition of another factor results in a 


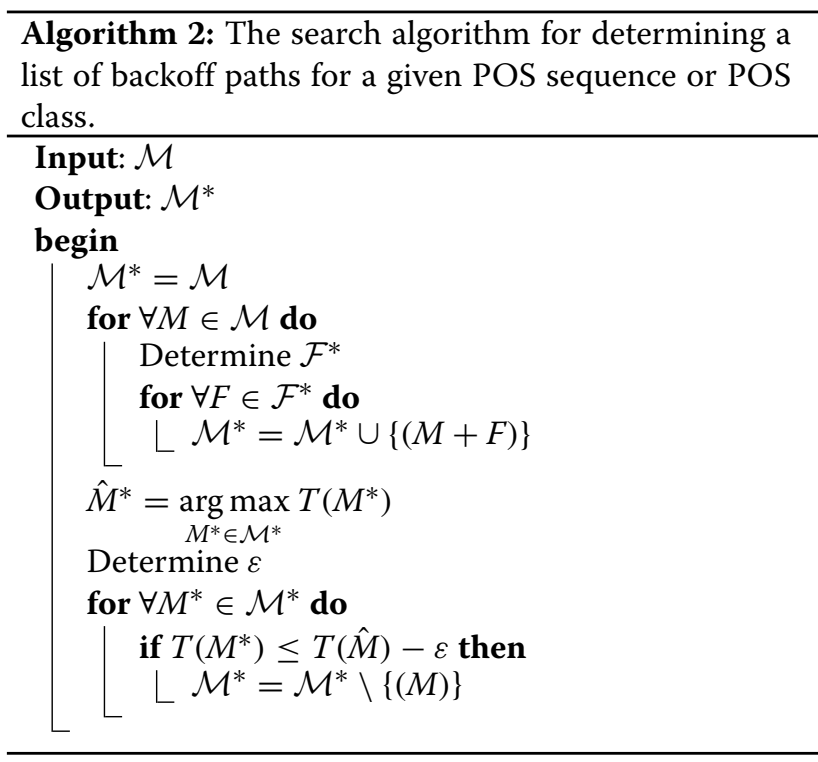

larger change of perplexity than in longer paths. If we expand shorter paths with a certain difference in performance with additional factors, the worse performing path has a greater chance of being expanded into a path that will later outperform other paths.

The described search algorithm is executed for a given class of POS sequences, and it must be repeated for every class. These classes will be defined in the next section.

\subsection{POS sequence classes}

Due to the large number of POS sequences, data sparsity will become inevitable. We decided to group POS sequences into a smaller number of classes based on their similarity. Each class will consist of one or more POS sequences, and the final application will make use of a backoff path assigned to the class containing a certain POS sequence. Thus, the criterion function and the search algorithm described above will be performed using classes of POS sequences.

Given that the primary problem leading to the need for POS classes is data sparsity for some POS sequences, we decided that the algorithm shall merge the POS class with the smallest number of occurrences in the training set with the most similar POS class. The final result shall be a set of POS classes that all have a sufficient number of occurrences and contain similar POS sequences. The algorithm takes a set of classes as input and returns a smaller set of classes as output.

We define the first set of classes with one set for each POS sequence that has occurred. The merging algorithm then performs several iterations to reduce the number of classes until it reaches the outer loop threshold $\alpha$.
In these iterations, we first determine a list of possible backoff paths for each class using the search algorithm described above. We then perform several iterations of class merging, where the class with the smallest number of occurrences $\bar{P}^{\prime}$ is merged with the most similar class $\bar{P}^{\prime \prime}$. For this purpose, we need to define the similarity function $D$. We repeat the merging until the number of classes falls below the inner loop threshold $\beta$.

The similarity between two classes is based on the two obtained lists of backoff paths. We look for a backoff path that gives good (but not necessarily the best) results in both classes. When faced with a small number of occurrences, we can also incorporate the POS sequences themselves to determine the similarity of classes. Other information can also be included to determine the similarity between classes.

The algorithm was conceived with two nested loops. The outer loop determines the final number of classes and incorporates the inner loop for a trade-off between speed and reliability. We might assume that if two classes are similar, they will also be similar to the merged class. We do not assume that after several iterations, merged classes will still behave similar to the original classes from which they were merged; we therefore incorporated an inner loop that determines how many merging iterations we want to perform before determining new backoff path lists for the merged classes.

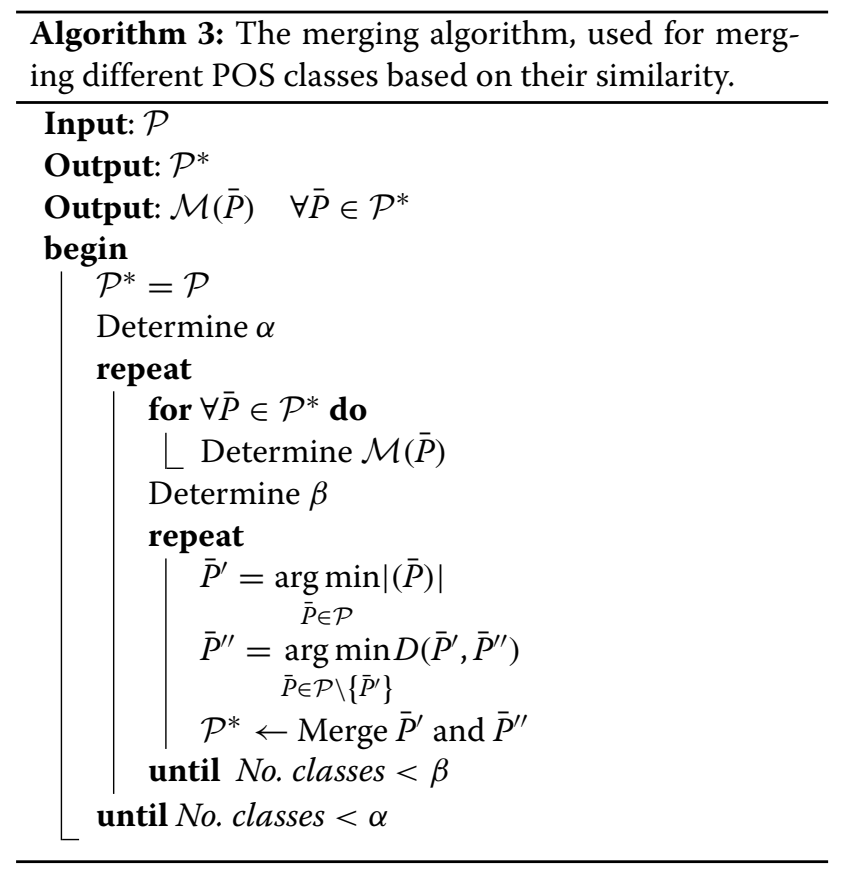

\subsection{Final path selection}

After reducing the number of POS classes to $\alpha$ using the merging algorithm, we perform a final run of the search 
algorithm. The output will again be a list of different backoff paths. For the final solution, one of them must be selected.

One simple solution would be to choose the model with the smallest perplexity, regardless of other considerations. However, we can compare the models by their perplexity as well as their size. We are therefore able to make a tradeoff between the expected results and the expected speed of the models.

We propose a simple algorithm to select another model, where we first consider smaller models that would estimate probabilities faster. We first choose the smallest model. We then test all other models in ascending order of model size. If a larger model has a lower result of the criterion function $T$ (i.e., higher perplexity), we skip it. If a larger model has a significantly higher result of the criterion function, we select this model as our current choice. We need to determine a threshold $\gamma$ that defines how much better the result of the criterion function must be in order to call it significant.

We do not want to select a significantly larger model if the criterion function increases only by a minimal amount; however, we still want to allow the possibility of choosing a slightly larger model. Therefore, we allowed the selection of a larger model if the criterion function gives a better result and the model's size increases by an amount smaller than the threshold $\delta$.

The process of selecting the final model is represented in Algorithm 4. We are again forced to determine two heuristic constants: $\gamma$ and $\delta$. We can start by setting $\gamma=0$ and $\delta=\infty$ so that the algorithm will give us the model with the best criterion function result. We can then change the values of both constants to achieve a compromise between performance and speed.

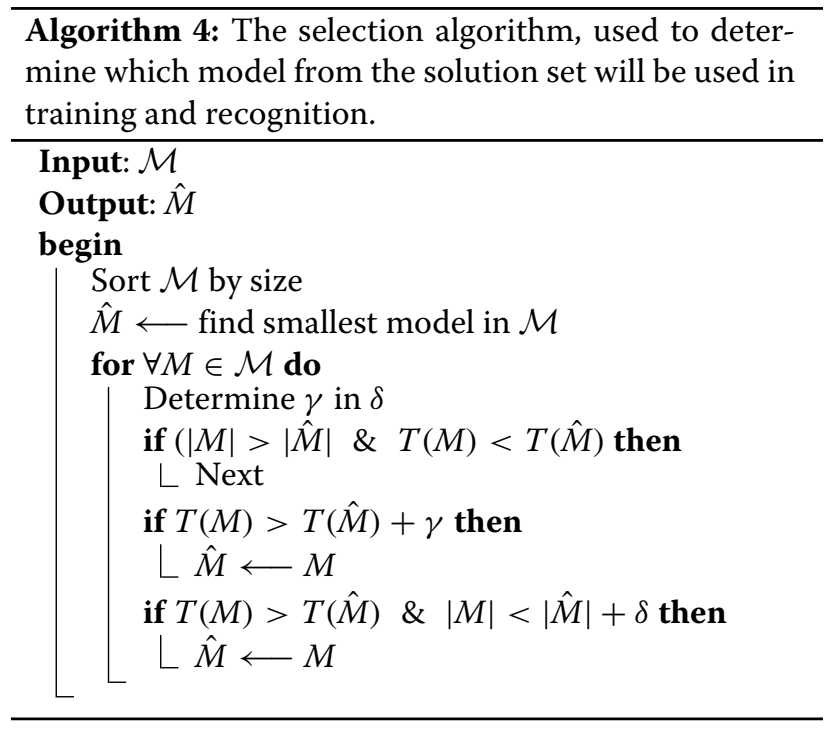

\subsection{Model usage}

Our final result obtained by the selection algorithm is the backoff function. This function is determined for each model order and each defined factor. While using a context-dependent FLM for a given word sequence, we decide which model order we want to use. Usually, it would be the largest order available, except at the beginnings of speech segments, where there are fewer words in the context. We then determine the POS sequence and look up the POS class in which this sequence has been merged. For each factor, we find the selected path for this function and then calculate estimates using these backoff paths.

\section{The recognition algorithm}

An appropriate system for testing the performance of context-dependent models is a two-pass continuous speech recognition algorithm.

In the first recognition pass, we typically make use of acoustic models and word-based language models. The main requirement of the speech recognition search algorithm is that it will produce a list of hypotheses for each speech segment. In the first pass, each hypothesis $W$ is scored with the equation

$$
P(W)=p_{A} P_{A}(W)+p_{L} P_{L}(W)+p_{I} C(W),
$$

where $P_{A}$ and $P_{L}$ are probabilities obtained with an acoustic and a word-based language model, respectively. Function $C$ is a simple word count function. We call these results the individual scores. Coefficients $p_{A}, p_{L}$, and $p_{I}$ are the acoustic model weight, the language model weight, and the word insertion penalty, respectively.

We then use an MSD tagger to annotate the hypotheses with all information used in the context-dependent FLMs. In order to reduce the space requirement for the hypotheses, we shall include only useful information, e.g., we can discard information produced by MSD taggers, which will not be used by the models.

In the second recognition pass, the hypotheses are rescored. We can use all three scores from the first pass and add scores from context-dependent FLMs. For example, if we have three factors, i.e., three FLMs, the scoring function becomes

$$
\begin{aligned}
P(W)= & p_{A} P_{A}(W)+p_{L} P_{L}(W)+p_{I} C(W) \\
& +p_{1} P_{f 1}(W)+p_{2} P_{f 2}(W)+p_{3} P_{f 3}(W),
\end{aligned}
$$

where $P_{f 1}(W), P_{f 2}(W)$, and $P_{f 3}(W)$ are probability scores obtained by the three context-dependent FLMs. Each of these three scores also has an appropriate weight: $p_{1}, p_{2}$, and $p_{3}$.

In both recognition passes, we have to optimize all weights using a development set. Usually, we set the 
acoustic model weight to 1 and we use an optimization algorithm for the remaining weights.

We perform several recognition run-throughs on the development set to find the best language model weight and word insertion penalty for the first recognition pass. We then use the optimized values in a single recognition run-through on the evaluation set.

In the second pass, we use lists of $N$-best hypotheses from both the development and the evaluation sets. We optimized all model weights and the word insertion penalty again on the development set and used them in the evaluation set.

A brute-force exhaustive search of optimal values would again not be feasible, as we have several real-valued values to optimize. Two weights are already present in the first pass but need to be optimized again. For each factor in the context-dependent FLMs, we have an additional weight to optimize. A simple algorithm was devised to optimize these weights. The algorithm works on $N$-best lists of all hypotheses in the development set. It starts with an initial set of values for all weights and then optimizes only one weight at a time while all other weights remain constant, before moving on to the next weight. The algorithm performs several iterations of optimizing all weights for a predefined number of times or until the optimized values do not differ from the values obtained in the previous iteration. We can repeat the algorithm with different starting values.

\section{Experimental system}

We evaluated the performance of the proposed language models on a large vocabulary continuous speech recognition (LVCSR) application in an inflective language, namely a Slovene Broadcast News transcription. The general structure of our system is presented in Fig. 4.

\subsection{Speech databases}

The Slovene Broadcast News (BNSI) database [21] was the only speech database used; it is also the only Slovene speech database appropriate for LVCSR. It consists of approximately $25 \mathrm{~h}$ of transcribed speech data. The largest part serves for acoustic model training, while the rest makes up the development set and the evaluation set, each consisting of approximately $2.5 \mathrm{~h}$ of speech data. We used the development set in all parameter optimization processes and the evaluation set for obtaining recognition results.

We trained word-based $n$-gram language models on the 620 million words FidaPLUS corpus of the Slovene language [22], which consists mainly of articles from newspapers and journals. While it is available in a lemmatized and MSD-tagged form, we used only basic word forms from it.

The Slovene ssj500k corpus is a manually MSDtagged and lemmatized corpus consisting of approximately 500,000 words. We used it for the training of all FLMs. It is large enough to effectively train models with a limited vocabulary-in this case POS tags and MSD tags.

The MSD tags in the ssj500k corpus follow the JOS specifications [18], which themselves were derived from the MULTEXT-EAST system [17]. Depending on the POS tags, the MSD tags can hold several grammatical categories. However, we found that only a few are useful in the presented application: POS, type, gender, case, number, and person. All other tags were removed.

\subsection{First pass}

The speech database was manually segmented. The trained acoustic models were HMM triphones with 16 Gaussian mixtures. We used 39 features of mel-frequency cepstral coefficients (MFCC): energy features and 12 features with delta and delta-delta coefficients.

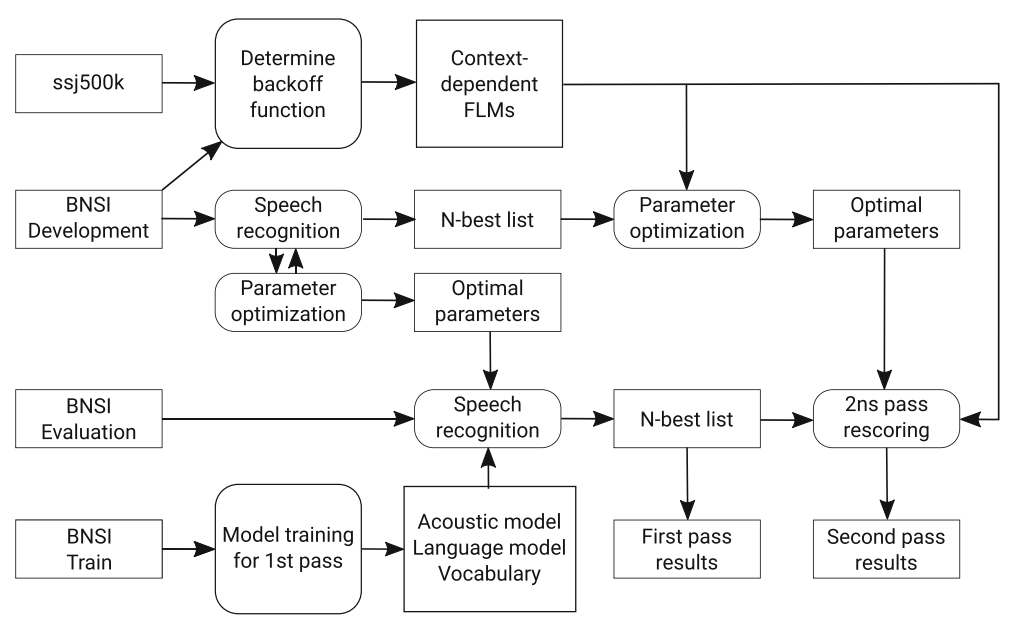

Fig. 4 The experimental system 
The vocabularies in the first pass include the most common words in the FidaPLUS corpus. We built vocabularies ranging from 60,000 to 300,000 words, constructing bigram and trigram models on all vocabulary sizes. Two smoothing techniques were used: Good-Turing and modified Knesser-Ney.

In the first pass, we used a Viterbi decoder to obtain an $N$-best list for the development and evaluation sets $(N=1000)$. We repeated the recognition on the development set in order to obtain optimal parameters for the language model weight and the word insertion penalty and used the optimized values on the evaluation set. We used the Obeliks tagger-an MSD tagger developed especially for the Slovene language-on all $N$-best lists.

\subsection{Context-dependent model training}

The first step in building context-dependent models was to define factors. The Obeliks tagger generates tags according to the JOS specifications, in which 12 different POS tags are defined, some of them having different types. Combining the POS tag and type, we defined an extended POS tag, e.g., we distinguished the types common noun and proper name instead of using noun. We decided to use extended POS tags due to grammatical concerns, as different types of the same part of speech can have different grammatical properties. The extended POS tags had 32 possible values. According to the search space estimation in the previous section, we have a total number of over one billion possible POS sequences with lengths up to six.

Next, we built MSD tags that included only gender, case, number, and person. Preliminary experiments showed that those grammatical categories could improve performance in speech recognition, while other grammatical categories cannot. We therefore used a reduced MSD tag set. Different parts of speech can have from 1 to 255 different values of the reduced MSD tag. The last chosen factor was the word itself.

We trained all MSD models on the ssj500k corpus. Not all possible POS sequences appeared in the corpus. For the first determination of POS sequence classes, we used only those sequences that did appear in the corpus. In Table 1, the number of classes depending on the model order is shown. The numbers are significantly lower than the theoretical upper limit, as most theoretically possible sequences do not produce meaningful or grammatically sensible sentences.

While implementing all of the necessary algorithms to determine the backoff function, we had to determine several heuristic constants. We did this experimentally to achieve good performance with reasonable time and space demands. The beam width $\varepsilon$ itself is newer fixed but is rather determined based on the currently best hypotheses in the search algorithm and the number of factors already in the backoff path, e.g., if the path has perplexity
Table 1 Number of POS classes given the model order $n$ observed in training and used for the first determination of POS classes

\begin{tabular}{ll}
\hline$n$ & Number of sequences \\
\hline 1 & 30 \\
2 & 475 \\
3 & 3003 \\
4 & 8666 \\
5 & 13,682 \\
6 & 15,478 \\
\hline
\end{tabular}

$P P_{\min }$ and a certain number of factors, the beam width is determined as

$$
\varepsilon=P P_{\min } \cdot F_{\varepsilon},
$$

where values for $F_{\varepsilon}$ are given in Table 2. Any backoff path with a perplexity higher than $P P_{\min }+\varepsilon$ will be discarded. Additionally, we implemented further criteria in the pruning procedure. If the set of possible backoff paths contains two paths that contain the same factors in a different order, we excluded the worse performing model. We found that the search algorithm time demands changed by about $10 \%$, when using different values of the factors in Table 2 ranging from 0.1 to 2 at all path lengths and, therefore, different values for $\varepsilon$. Finally, the values in Table 2 were chosen based on preliminary results obtained during the implementation of the search algorithm.

Unlike $\varepsilon$, the thresholds $\alpha$ and $\beta$ in the merging algorithm have almost no impact on the time demands in determining the backoff function.

We determined $\alpha=10$ for unigram models and $\alpha=50$ for higher order models to be suitable values. The parameter $\beta$ is determined in each iteration at run-time. We added the constraint that the number of classes in the inner loop must not be reduced by more than $50 \%$.

Lastly, we had to determine the parameters $\gamma$ and $\delta$ in the selection algorithm. Those parameters have no effect on time demands for training. It was also found that they do not have a significant impact on model performance. We chose $\gamma$ to be $5 \%$ of the perplexity of the currently

Table 2 Pruning width factors $k$ for the search algorithm given the number of factors

\begin{tabular}{lc}
\hline No. factors & $F_{\varepsilon}$ \\
\hline 1 & 1.0 \\
2 & 0.5 \\
3 & 0.3 \\
4 & 0.2 \\
$\geq 5$ & 0.1 \\
\hline
\end{tabular}


selected model and $\delta$ to be $25 \%$ of the size of the currently selected model in terms of entries in the language model.

\subsection{Computational demands}

The total time to perform the search algorithm was $56 \mathrm{~h}$, of which the largest part $(36 \mathrm{~h})$ was spend using model order $n=5$. It was performed on a dual-processor server with 88 logical threads at $3.6 \mathrm{GHz}$ and $128 \mathrm{~GB}$ of memory. We shall note that the exact time depends not only on hardware configuration but also on software, other server load, development and training sets, and the possibility of parallel processing. We also reused already build models.

Rather than comparing real-time values, we shall state the number of trained models. Table 3 shows the number of all possible backoff paths given the model order $n$ and the current factor $k$. The number of maximum models is based on Eq. 5; however, we also limited the backoff path length to eight factors. We also show the number of models that were actually trained and tested with our final search algorithm. We see that at higher model order, the number of tested models only slightly increases, while the number of maximum models reaches infeasible numbers. The final ratio between the sum of all tested and sum of all possible models is 1:1100. This means that we tested 1100 times fewer models than we would have to in brute force. Even, if we consider models with an order of up to 4, this ratio would be 1:200.

Table 3 Comparison of the number of all possible backoff paths with the number of trained and tested paths with regard to model order $n$ and modeled factor $k$

\begin{tabular}{llll}
\hline$n$ & $k$ & Maximum & Tested \\
\hline 1 & 1 & 1 & 0 \\
1 & 2 & 2 & 1 \\
1 & 3 & 5 & 4 \\
2 & 1 & 16 & 15 \\
2 & 2 & 65 & 55 \\
2 & 3 & 326 & 176 \\
3 & 1 & 1957 & 705 \\
3 & 2 & 13,700 & 1758 \\
3 & 3 & 109,601 & 5129 \\
4 & 1 & 623,530 & 15,320 \\
4 & 2 & $2.61 \times 10^{6}$ & 15,505 \\
4 & 3 & $8.71 \times 10^{6}$ & 21,552 \\
5 & 1 & $2.47 \times 10^{7}$ & 56,888 \\
5 & 2 & $6.19 \times 10^{7}$ & 45,085 \\
5 & 3 & $1.41 \times 10^{8}$ & 51,466 \\
& & $2.40 \times 10^{8}$ & 213,659 \\
\hline
\end{tabular}

It should be noted that one possibility is a model without any factors. This model was not tested as it always gives the lowest performance. Therefore, the number of all possible backoff paths in the table is 1 higher than the number of tested models at data points where otherwise all models were tested.

\subsection{Simple MSD models}

In order to determine whether the context-dependent backoff paths have any effect on the recognition performance that is not due to the inclusion of MSD data itself, we also built simple language models based on MSD tags. These models are based on the extended POS tag $(P)$ and the MSD tag $(M)$.

The first set of models is defined by

$$
\begin{aligned}
& P\left(P_{0} \mid P_{-1}, \ldots, P_{-n}\right), \\
& P\left(M_{0} \mid M_{-1}, \ldots, M_{-n}\right) .
\end{aligned}
$$

The backoff path in both models is to remove the most distant factor. In the first set, we only use factors of the same type in the model. These models are $n$-gram models of extended POS tags and $n$-gram models of MSD tags. The second set of models is defined by

$$
\begin{aligned}
& P\left(P_{0} \mid P_{-1}, \ldots, P_{-n}, M_{0}, \ldots, M_{-n}\right), \\
& P\left(M_{0} \mid M_{-1}, \ldots, M_{-n}, P_{-1}, \ldots, P_{-n}\right) .
\end{aligned}
$$

Here, both types of factors are used in the same model. We built models with a maximum of seven conditional factors and considered at most five words of history. Therefore, we obtained 6-gram FLMs with fixed backoff paths of no more than eight vertices. The limits of the number of factors and history length are due to preliminary results indicating that longer paths are of no further benefit to the performance. Additionally, we added the constraint that if a particular factor appears in these models, then all factors of the same type that are closer to the modeled word must also appear.

Backoff paths are built simply by adding one of two possible next factors to the path. A total of 244 possible models were built, all with backoff paths that comply with the presented constraints.

These models were later used to obtain results regarding the degree to which performance can be improved if morphological information is introduced into the speech recognition algorithm without introducing context-dependent models.

\subsection{Tools}

We used the HTK toolkit with the speech decoder HDecode in acoustic model training and the first recognition pass. In order to build the language models (including FLMs), as well as for hypothesis rescoring, we used the SRILM toolkit, as it supports FLMs [23]. For backoff path 
determination and the second recognition pass, tools were developed within the framework of the present research.

\section{Results}

\subsection{First pass and $\mathbf{N}$-best lists}

Table 4 shows recognition results in word error rate (WER) for the first recognition pass with bigram and trigram language models with Good-Turing and modified Knesser-Ney smoothing and different vocabulary sizes. Vocabulary size and model order have an expected impact on performance. However, while other research indicated that modified Knesser-Ney smoothing performs better than Good-Turing [24], our results show the opposite, although there are only slight, statistically non-significant differences. The best WER result of $22.64 \%$ was obtained with a trigram Good-Turing smoothed model built on a $300 \mathrm{~K}$ vocabulary. Similar results can be obtained on the BNSI development set.

We can also compare computational and memory consumption. Table 5 shows real-time factors (RTF) for all first-pass scenarios. We notice only small differences with regard to the smoothing method. Results show that RTF increases by a factor of 2 if the vocabulary size is increased from $60 \mathrm{~K}$ to $300 \mathrm{~K}$ and by a factor of 3 if when using trigram models compared to bigram models. Considering also recognition results, we see that the increase in vocabulary brought a larger performance increase with a smaller RTF increase than the increase in model order.

However, increasing the vocabulary size mainly eliminates errors due to out-of-vocabulary words. With the $300 \mathrm{~K}$ vocabulary, the out-of-vocabulary rate is $1.02 \%$ and further enlargement of the vocabulary results in only small recognition improvements.

Preliminary research showed us that there are also only small differences in model perplexity if we increase the model order to 4-gram models. Also, the recognition results we found increase only slightly if at all. This was found using a two-pass rescoring algorithm as the recognition tool (HDecode) supports only bigram and trigram models.

Table 6 shows us the the memory demands for all firstpass scenarios. We can again observe that increase in

Table 4 Recognition results in the first pass obtained on the BNSI evaluation set

\begin{tabular}{|c|c|c|c|c|}
\hline \multirow{3}{*}{ Vocabulary size } & \multicolumn{4}{|l|}{ WER [\%] } \\
\hline & \multicolumn{2}{|c|}{ Good-Turing } & \multicolumn{2}{|c|}{ Knesser-Ney } \\
\hline & Bigram & Trigram & Bigram & Trigram \\
\hline $60 \mathrm{~K}$ & 33.89 & 30.73 & 33.84 & 30.95 \\
\hline $100 \mathrm{~K}$ & 31.27 & 28.08 & 31.24 & 28.36 \\
\hline $200 K$ & 29.70 & 26.27 & 29.78 & 26.53 \\
\hline $300 K$ & 29.22 & 25.64 & 29.28 & 25.87 \\
\hline
\end{tabular}

Table 5 Average real-time factors for recognition in the first pass obtained on the BNSI evaluation set

\begin{tabular}{|c|c|c|c|c|}
\hline \multirow{3}{*}{ Vocabulary size } & \multicolumn{4}{|c|}{ Real-time factor } \\
\hline & \multicolumn{2}{|c|}{ Good-Turing } & \multicolumn{2}{|c|}{ Knesser-Ney } \\
\hline & Bigram & Trigram & Bigram & Trigram \\
\hline $60 \mathrm{~K}$ & 6.29 & 18.46 & 6.14 & 18.62 \\
\hline $100 \mathrm{~K}$ & 7.82 & 23.42 & 7.52 & 23.37 \\
\hline $200 \mathrm{~K}$ & 10.44 & 31.55 & 10.48 & 31.71 \\
\hline $300 K$ & 12.66 & 37.09 & 12.88 & 37.70 \\
\hline
\end{tabular}

vocabulary size increases the memory demands slightly less than the increase in model order.

We have to add that the exact values for RTF as well as memory consumption depended on the computer hardware and the software tools used.

When rescoring the $N$-best list, there is always an upper limit of how much improvement can be achieved, as only a limited number of hypotheses are available in the second pass. Assuming we had a system in the second pass that would always select the best hypothesis (an oracle), we would still have an error rate-the so-called oracle error rate (OER) - that is dependent on the number of hypotheses. The OER results on the BNSI evaluation set using bigram and trigram language Good-Turing models are shown in Figs. 5 and 6, respectively. Similar OER results can also be obtained for other models and on the BNSI development set. In both figures, we can see that the oracle error rate at 1000 hypotheses is approximately $50 \%$ lower than the WER.

We tagged the hypotheses obtained with the bigram and trigram Good-Turing models with the $300 \mathrm{~K}$ vocabulary for use in the second recognition pass. It was decided to use the $N$-best lists with the best WER results, as well as the list obtained with the corresponding bigram model. The used tagger is rather slow and had real-time factors from 26.07 to 26.67 .

\subsection{Context-dependent models}

Several models were tested in the process of determining the backoff functions for different maximal model orders

Table 6 Peak memory usage in the first pass obtained on the BNSI evaluation set

\begin{tabular}{|c|c|c|c|c|}
\hline \multirow{3}{*}{ Vocabulary size } & \multicolumn{4}{|c|}{ Memory usage [MB] } \\
\hline & \multicolumn{2}{|c|}{ Good-Turing } & \multicolumn{2}{|c|}{ Knesser-Ney } \\
\hline & Bigram & Trigram & Bigram & Trigram \\
\hline $60 \mathrm{~K}$ & 743 & 1287 & 745 & 1299 \\
\hline $100 \mathrm{~K}$ & 917 & 1512 & 914 & 1530 \\
\hline $200 K$ & 973 & 1776 & 1110 & 1815 \\
\hline $300 K$ & 1252 & 1934 & 1273 & 1935 \\
\hline
\end{tabular}




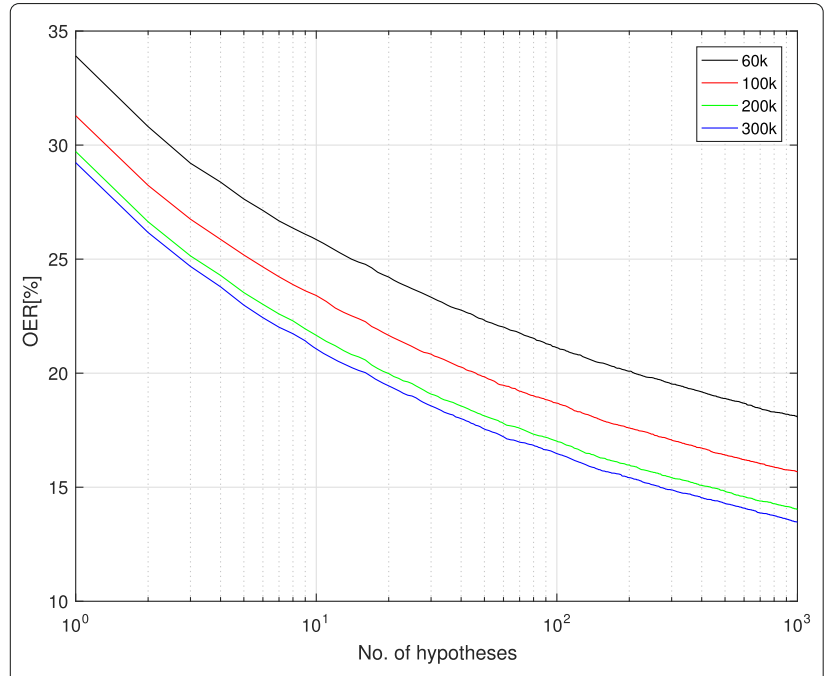

Fig. 5 Oracle error rate on the BNSI evaluation set with bigram models and different vocabulary sizes

and different factors. The total number of tested models was 407,892 , of which 322 were in the final selection for all backoff functions. In all cases, the number of selected models in a single backoff function lies below the threshold in the merging algorithm, which defines the maximum number of classes. The number is lower because some classes use the same backoff path as others.

Table 7 shows the perplexity results of contextdependent FLMs on the evaluation set. These perplexities were obtained using the backoff function for probability estimations in the text.

We separately rescored the $N$-best lists obtained with a bigram and a trigram model in the first pass. The results

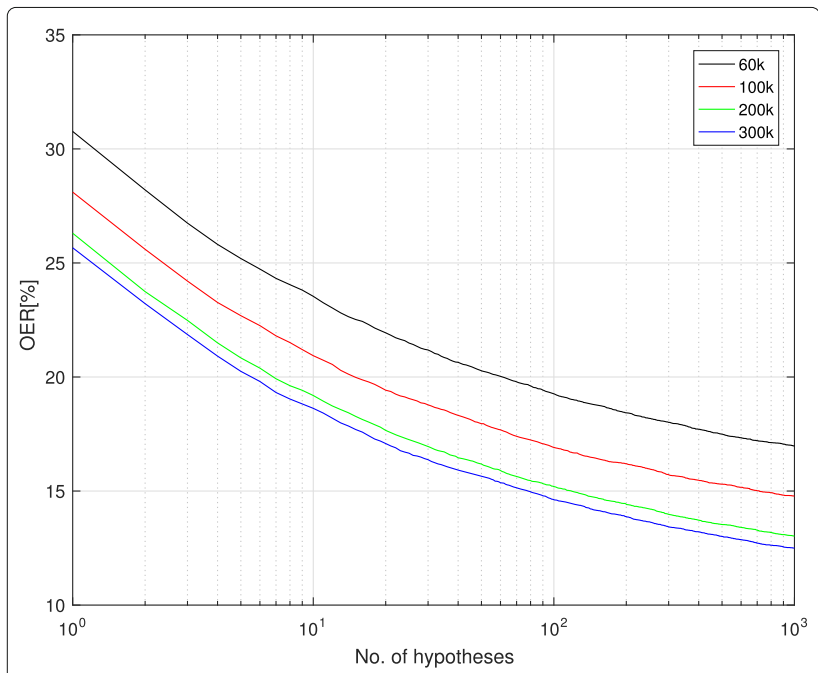

Fig. 6 Oracle error rate on the BNSI evaluation set with trigram models and different vocabulary sizes
Table 7 Perplexity results of context-dependent FLM on the BNSI development set

\begin{tabular}{llll}
\hline \multirow{2}{*}{ Model order } & \multicolumn{3}{l}{ Modeled factor } \\
\cline { 2 - 4 } & $P$ & $M$ & $W$ \\
\hline 1 & $/$ & 6581 & 38,867 \\
2 & 8392 & 3490 & 33,536 \\
3 & 7275 & 3147 & 31,294 \\
4 & 6561 & 2970 & 29,447 \\
5 & 6231 & 2876 & 27,947 \\
\hline
\end{tabular}

are shown in Tables 8 and 9, respectively. We repeated rescoring using any one, any two, or all three FLMs. We also indicate the model order with which the best results were obtained.

The results show significant increases in performance when bigram language models were used in the first pass. We also used a traditional trigram language model in the second pass instead of the bigram model from the first pass. Thus, part of the increased performance is due to this model and part to the use of context-dependent FLM. We decided to do so as the use of bigram models in the first pass is much faster and the results can be largely improved with traditional trigram language models in the second pass.

Still, the results do now outperform the use of trigram language models in the first pass. The best improvement is observed using only the FLM for MSD tags. Furthermore, combinations with this model show greater improvement than other combinations.

When rescoring the results obtained with a trigram model in the first pass, the differences are smaller as the same traditional language model was used. However, in this case, we can see the impact that context-dependent FLMs have on performance. When only one FLM is used, the best improvement is obtained with the model for MSD tags. Using the model for POS tags even lowers the results. This can be due to either the nature of this model or the differences in the evaluation and developments on the basis of which the parameters were optimized. We also

Table 8 Recognition results on the evaluation set using bigram models in the first pass and context-dependent models in the second pass

\begin{tabular}{llll}
\hline FLMs & Optimal model order & WER [\%] & Relative improvement[\%] \\
\hline$P$ & 5 & 26.51 & 9.26 \\
$M$ & 4 & 25.84 & 11.56 \\
$W$ & 5 & 26.07 & 10.78 \\
$P+M$ & 5 & 25.92 & 11.27 \\
$P+W$ & 5 & 26.38 & 9.71 \\
$M+W$ & 4 & 25.92 & 11.30 \\
All & 5 & 26.01 & 10.99 \\
\hline
\end{tabular}


Table 9 Recognition results on the evaluation set using trigram models in the first pass and context-dependent models in the second pass

\begin{tabular}{llll}
\hline FLMs & Optimal model order & WER [\%] & Relative improvement[\%] \\
\hline$P$ & 3 & 25.90 & -1.01 \\
$M$ & 5 & 25.39 & 0.99 \\
$W$ & 5 & 25.46 & 0.70 \\
$P+M$ & 5 & 25.61 & 0.14 \\
$P+W$ & 5 & 25.95 & -1.20 \\
$M+W$ & 2 & 25.20 & 1.73 \\
All & 5 & 25.45 & 0.74 \\
\hline
\end{tabular}

see that combinations using the POS tag FLM show worse results. The best results are obtained using models for the MSD tag and for the basic word form. We observe a 1.73\% relative improvement over the first-pass result.

The results in both tables suggest that POS tag FLMs are not beneficial to recognition accuracy, while both of the other models are.

The computational and memory demands in the second pass are much smaller, as only language models are used on a finite number of sentences. The real-time factor in the second pass is about 0.50 , while the real-time factor for simple models is about 0.01 .

\subsection{Comparison with simple MSD models}

We performed the same two-pass algorithm and parameter optimization using simple MSD models. The recognition results after the second pass on the evaluation set are shown in Table 10. Results are shown for using either bigram or trigram language models in the first pass and either only the model for MSD tags or both models for MSD and POS tags in the second pass.

The results using bigram models in the first pass are comparable with the use of context-dependent models. The results obtained while using trigram models in the first pass show that recognition accuracy was improved only by $0.07 \%$ when using both models, for MSD tags and POS tags. The accuracy was even reduced while using only the model for MSD tags. Results using only the model for POS tags were significantly lower.

Table 10 Recognition results on the evaluation set using simple MSD models for POS tags $(P)$ and MSD tags (M)

\begin{tabular}{llll}
\hline $\begin{array}{l}\text { First pass } \\
\text { model }\end{array}$ & $\begin{array}{l}\text { Second pass } \\
\text { model }\end{array}$ & WER [\%] & $\begin{array}{l}\text { Relative } \\
\text { improvement[\%] }\end{array}$ \\
\hline Bigram & $M$ & 25.84 & +11.56 \\
Bigram & $M+P$ & 26.01 & +10.99 \\
Trigram & $M$ & 25.86 & -0.84 \\
Trigram & $M+P$ & 25.62 & +0.07 \\
\hline
\end{tabular}

The comparison shows that the simple addition of grammatical information does not improve the recognition results significantly unless we use this information in a more sophisticated model.

\subsection{Error analysis}

We analysed the best results in the first pass and their best improvement in the second pass in more detail.

The statistical significance of the improvement was tested with the approximate randomization test. This test was selected because it does not require special assumptions about the evaluation set. With 10,000 runs, we obtained a $p$ value of 0.0055 , indicating that the results were statistically significant at a significance threshold of 0.01 .

Table 11 shows the basic results of inserted, deleted, and substituted words. The largest difference can be seen in the number of deleted words-a reduction of $16 \%$ relatively - while other types of error increased slightly.

Table 12 shows the WER results regarding different parts of speech. The results are shown for the first and second pass, displaying the difference as well as values relative to the number of occurrences in the reference transcription. The greatest improvements occur with prepositions and conjunctions. Considering these results and the data in Table 11, we can conclude that context-dependent FLMs have the most impact on correcting deletion errors involving short words, mainly minor parts of speech.

The results for inflected parts of speech are mixed. While the error rates for verbs, adjectives, and pronouns decreased, the error rates for nouns and numerals increased. Although the increase WER for nouns is rather small, the increase for numerals is more significant.

Finally, Table 13 shows the results for inflectional parts of speech, where the correct POS and the correct lemma but the false word form were recognized. These errors indicate falsely recognized word endings. The percentage values are given relative to the total number of substitution errors of the POS, e.g., 866 nouns were substituted with other nouns, while 296 of these substitutions (34.58\%) were with a noun with the same lemma. We see that approximately $40 \%$ of all errors on inflectional parts of speech are due to falsely recognized word forms. In

Table 11 Comparison of recognition errors by type between the first and second pass

\begin{tabular}{llll}
\hline Error type & 1st pass & 2nd pass & Difference \\
\hline Deletion & 1446 & 1216 & -230 \\
Insertion & 401 & 498 & 97 \\
Substitution & 4013 & 4043 & 30 \\
Total & 5860 & 5757 & -103 \\
\hline
\end{tabular}


Table 12 Comparison of recognition errors by POS between the first and second pass

\begin{tabular}{lllll}
\hline POS & Total & 1st pass & 2nd pass & Difference \\
\hline Noun & 6721 & $23.20 \%$ & $23.26 \%$ & $+0.25 \%$ \\
Verb & 3901 & $28.22 \%$ & $27.66 \%$ & $-2.00 \%$ \\
Adjective & 2646 & $20.67 \%$ & $20.33 \%$ & $-1.65 \%$ \\
Adverb & 1667 & $24.84 \%$ & $24.18 \%$ & $-2.66 \%$ \\
Pronoun & 1536 & $34.18 \%$ & $33.27 \%$ & $-2.67 \%$ \\
Numeral & 747 & $27.31 \%$ & $27.71 \%$ & $+1.47 \%$ \\
Preposition & 2499 & $27.53 \%$ & $26.65 \%$ & $-3.19 \%$ \\
Conjunction & 1964 & $28.16 \%$ & $26.78 \%$ & $-4.88 \%$ \\
Particle & 1038 & $24.76 \%$ & $24.47 \%$ & $-1.17 \%$ \\
Interjection & 8 & $100.00 \%$ & $50.00 \%$ & $-50.00 \%$ \\
Residual & 6 & $66.67 \%$ & $100.00 \%$ & $+50.00 \%$ \\
\hline
\end{tabular}

the second pass, the total number of errors decreased by $1.65 \%$ relatively.

\section{Conclusions}

In the present paper, we proposed a new method of determining the backoff path for factored language models. As the basic method leads to data sparsity and a large search space, we also presented all of the necessary algorithms to build a feasible system. We demonstrated that models using these backoff paths outperformed factored language models using more simply determined backoff paths. We also demonstrated that simpler models might not have any potential to improve recognition results, while the use of models with context-dependent backoff paths resulted in an accuracy improvement that was shown to be statistically significant.

The methods presented still leave room for the further optimization of several parameters used in the algorithms. Training set and factor selection may also be considered in further research. While we performed the experiments on Slovene, the proposed models can also be used in other inflectional or otherwise morphologically complex

Table 13 Falsely recognized word forms of inflectional parts of speech relative to the total number of substitutions within the same part of speech

\begin{tabular}{lllll}
\hline POS & \multicolumn{2}{c}{ 1st pass } & \multicolumn{2}{c}{ 2nd pass } \\
\hline Noun & 296 & $34.58 \%$ & 291 & $34.64 \%$ \\
Adjective & 135 & $57.94 \%$ & 126 & $53.58 \%$ \\
Verb & 183 & $48.93 \%$ & 177 & $47.58 \%$ \\
Pronoun & 47 & $52.22 \%$ & 46 & $51.11 \%$ \\
Numeral & 16 & $20.78 \%$ & 16 & $21.33 \%$ \\
Total & 667 & $41.53 \%$ & 656 & $40.72 \%$ \\
\hline
\end{tabular}

languages. Further research on the possibility of combining the presented statistics-based method with formal knowledge-based methods could also lead to improvements in recognition of word relations in a spoken sentence and consequently to a reduction in recognition errors.

\section{Acknowledgements}

This work was partially financially supported by the Slovenian Research Agency (ARRS) under contract number 1000-10-310131.

\section{Authors' contributions}

This paper shows results from the doctoral research of GD under the supervision of ZK. Both authors read and approved the final manuscript.

\section{Competing interests}

The authors declare that they have no competing interests.

Received: 13 October 2016 Accepted: 20 February 2017

Published online: 28 February 2017

\section{References}

1. S Zablotskiy, K Zablotskaya, W Minker, in Intelligent Environments (IE), 2010 Sixth International Conference on. Some approaches for Russian speech recognition, (Kuala Lumpur, 2010), pp. 96-99

2. TRotovnik, MS Maučec, Z Kačič, Large vocabulary continuous speech recognition of an inflected language using stems and endings. Speech Commun. 49(6), 437-452 (2007)

3. K Kirchhoff, D Vergyri, J Bilmes, K Duh, A Stolcke, Morphology-based language modeling for conversational Arabic speech recognition. Comput. Speech Lang. 20(4), 589-608 (2006)

4. AE Axelrod, Factored language models for statistical machine translation. (University of Edinburgh, 2006)

5. EM de Novais, Portuguese text generation using factored language models. J. Brazilian Comput. Soc. 19(2), 135-146 (2013)

6. K Kirchhoff, J Bilmes, K Duh, Factored language models tutorial. (University of Washington, 2016). http://ssli.ee.washington.edu/people/duh/papers/ flm-manual.pdf. Accessed 1 October

7. JA Bilmes, K Kirchhoff, in NAACL-Short '03. Factored language models and generalized parallel backoff, (Edmonton, 2003), pp. 4-6

8. M Lazăr, D Militaru, in Speech Technology and Human-Computer Dialogue (SpeD), 2013 7th Conference on. A Romanian language modeling using linguistic factors, (Cluj-Napoca, 2013), pp. 1-6

9. D Vazhenina, K Markov, in Awareness Science and Technology and Ubi-Media Computing (iCAST-UMEDIA), 2013 International Joint Conference on. Factored language modeling for Russian LVCSR, (Aizuwakamatsu, 2013), pp. 205-211

10. I Kipyatkova, A Karpov, in SPECOM 2014. Study of morphological factors of factored language models for Russian ASR, (Novi Sad, 2014), pp. $451-458$

11. H Sak, M Saraçlar, T Güngör, in ICASSP. Morphology-based and sub-word language modeling for Turkish speech recognition, (Dallas, 2010), pp. 5402-5405

12. MY Tachbelie, ST Abate, W Menzel, in Language and Technology Conference. Morpheme-based and factored language modeling for amharic speech recognition, (Poznaň, 2011), pp. 82-93

13. Z Alumae, in ICASSP. Sentence-adapted factored language model for transcribing estonian speech, (Toulouse, 2006), pp. 429-432

14. THirsimaki, J Pylkkonen, M Kurimo, Importance of High-Order N-Gram Models in Morph-Based Speech Recognition. IEEE Trans. Audio, Speech, Lang. Process. 17(4), 724-732 (2009)

15. AED Mousa, MAB Shaik, R Schlüter, H Ney, in INTERSPEECH. Morpheme based factored language models for German LVCSR, (Florence, 2011), pp. 1053-1056

16. H Adel, NT Vu, K Kirchhoff, D Telaar, T Schultz, Syntactic and Semantic Features For Code-Switching Factored Language Models. IEEE/ACM Trans. Audio, Speech, Lang. Process. 23(3), 431-440 (2015)

17. T Erjavec, in $L R E C$. Multext-east version 4: multilingual morphosyntactic specifications, lexicons and corpora, (Valletta, 2010), pp. 2544-2547 
18. T Erjavec, D Fišer, S Krek, N Ledinek, in LREC. The JOS Linguistically tagged corpus of Slovene, (Valletta, 2010), pp. 1806-1809

19. S Katz, Estimation of probabilities from sparse data for the language model component of a speech recognizer. IEEE Trans. Acoust. Speech Signal Process. 35(3), 400-401 (1987)

20. D Klakow, J Peters, Testing the correlation of word error rate and perplexity. Speech Commun. 38(1-2), 19-28 (2002)

21. A Žgank, D Verdonik, AZ Markušs, Z Kačič, in INTERSPEECH. BNSI Slovenian broadcast news database - speech and text corpus, (Lisbon, 2005), pp. 1537-1540

22. Š Arhar, V Gorjanc, S Krek, in Proceedings of the Corpus Linguistics Conference. FidaPLUS corpus of Slovenian. The new generation of the Slovenian reference corpus: its design and tools, (Birmingham, 2007)

23. A Stolcke, J Wheng, W Wang, V Abrash, in Proceedings IEEE Automatic Speech Recognition and Understanding Workshop. SRILM at sixteen: update and outlook, (Waikoloa, 2011)

24. SF Chen, J Goodman, An empirical study of smoothing techniques for language modeling. Comput. Speech Lang. 13(4), 359-394 (1999)

\section{Submit your manuscript to a SpringerOpen ${ }^{\circ}$ journal and benefit from:}

- Convenient online submission

- Rigorous peer review

- Immediate publication on acceptance

- Open access: articles freely available online

- High visibility within the field

- Retaining the copyright to your article

Submit your next manuscript at $\gg$ springeropen.com 